ARTICLE

\title{
Visualization of gaseous iodine adsorption on single zeolitic imidazolate framework-90 particles
}

Yuting Lei $i^{1}$, Guihua Zhang ${ }^{1}$, Qinglan Zhang ${ }^{1}$, Ling $\mathrm{Yu}^{1}$, Hua $\mathrm{Li}^{2}$, Haili Yu${ }^{1 凶} \& \mathrm{Yi} \mathrm{He}^{1 凶}$

Zeolitic imidazolate frameworks ( $\mathrm{ZIFs})$ are very useful as high-capacity iodine $\left(\mathrm{I}_{2}\right)$ adsorbents. The adsorption performance is usually probed by measuring a statistical average property over an entire sample consisting of a large number of ZIF particles, leaving the interparticle heterogeneity information among individuals. Here we report a dark-field microscopy (DFM) method to visualize gaseous $\mathrm{I}_{2}$ adsorption on single ZIF-90 particles in situ and in real time. The adsorption of $\mathrm{I}_{2}$ is found to alter the scattering spectrum of ZIF90 particles, inducing a distinct color change from bluewhite to yellow. According to correlating the adsorption amount of gaseous $I_{2}$ with the change of $B$ value from DFM images, we quantitatively image the adsorption process and estimate the related kinetic parameters at the single particle level. Single particle measurements clarify the large particle-to-particle heterogeneity in adsorption reactivity and significant adsorption activity improvement of ZIF90 after introduction of linker defects, which provides a microscopic understanding of the structure-activity relationship. We further demonstrate the capacity of this strategy for studying gaseous $I_{2}$ adsorption on single ZIF-91 particle as a derivative of ZIF-90 to illustrate the generality.

\footnotetext{
${ }^{1}$ National Collaborative Innovation Center for Nuclear Waste and Environmental Safety, School of National Defence Science \& Technology, Southwest University of Science and Technology, Mianyang, PR China. ${ }^{2}$ SUSTech Core Research Facilities, Southern University of Science and Technology, Shenzhen, PR

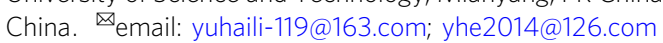


T he capture of gaseous iodine $\left(\mathrm{I}_{2}\right)$ has been attracting intense interest ${ }^{1,2}$. The gaseous $\mathrm{I}_{2}$ with a high mobility is the volatile product of uranium fission and iodide oxidation by atmospheric ozone ${ }^{3-5}$. It can cause radiation damage, air contamination, climate change, and atmospheric particle formation ${ }^{6-8}$, which constitutes a serious threat to the ecological environment and public health. The serious harm of gaseous $\mathrm{I}_{2}$ requires developing high-performance porous adsorbents for capturing it. As a metal-organic framework (MOF) subfamily, zeolitic imidazolate framework (ZIF) based on imidazolate and transition metal ion is one such material that is capable of trapping gaseous $I_{2}$ within the nanopore. The trapping capacity of ZIF for $\mathrm{I}_{2}$ is ascribed to the match in size and charge-transfer (CT) interaction between $\mathrm{I}_{2}$ molecule and aromatic imidazole ring ${ }^{9-11}$.

The majority of studies on the capture process of gaseous $\mathrm{I}_{2}$ by ZIF have relied on theoretical simulation, X-ray diffraction, and calorimetry. However, the structural heterogeneity among different ZIF particles in a sample is easily obscured by the aforementioned ensemble analysis methods. The properties and structures of individual particles are simply averaged in the ensemble sample consisting of billions of ZIF particles, which restricts the understanding of the structure-activity relationship. On the other hand, some attempts in the improvement of the capture of $\mathrm{I}_{2}$ confirm that it can amorphize ZIF-8 with distort pore apertures to kinetically trap $\mathrm{I}_{2}{ }^{12}$. Investigation on the dynamics of the reaction intermediate states is thus the key to clarify the kinetics, which is highly essential to design and develop active ZIF adsorbents. In order to obtain the kinetic, real-time monitoring the adsorption process of $\mathrm{I}_{2}$ at the single ZIF particle level is an important and challenging avenue in practice.

Here, we investigate the dynamic of the gaseous $\mathrm{I}_{2}$ adsorption on single ZIF-90 particles in situ and in real time with dark-field microscopy (DFM) (Fig. 1), a simple and powerful tool that can image individual nanoparticles with both high resolution and high contrast. Upon the introduction of gaseous $\mathrm{I}_{2}$, the B component of the DFM image of single ZIF-90 particles significantly decreases. This process accompanies distinct change in color and scattering peak, which enables and facilitates visual readout of the capture process of gaseous $\mathrm{I}_{2}$ by single ZIF-90 particle. By correlating the $\mathrm{B}$ value of the DMF image with adsorption amount of gaseous $I_{2}$, we qualitatively determine the diffusion coefficient of gaseous $\mathrm{I}_{2}$ within ZIF-90 at the single-particle level. We observe the quite heterogeneous adsorption capacity of different individual ZIF-90 particles. Furthermore, we will show that the introduction of more defects will greatly enhance the adsorption rate and amount of gaseous $\mathrm{I}_{2}$ on single ZIF-90 particles. Meanwhile, this method is appropriate for studying other ZIF particles such

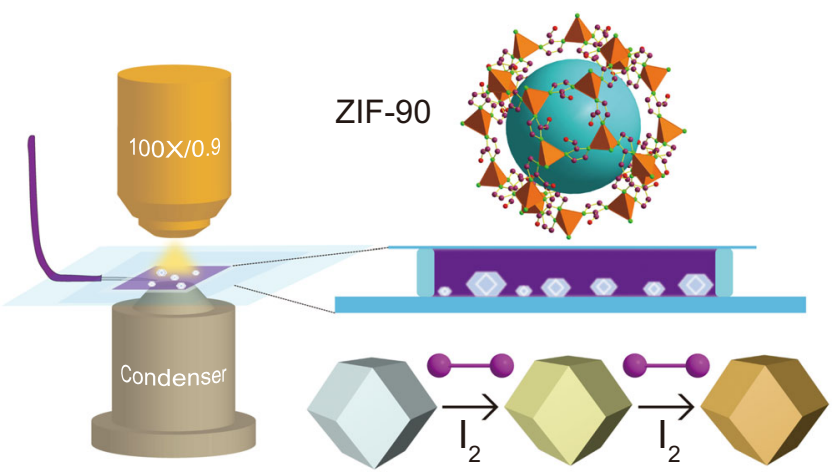

Fig. 1 Schematic illustration of visualizing gaseous $I_{2}$ adsorption on single ZIF-90 particles by dark-field microscopy. DFM setup, the structure of ZIF-90, microchamber, and the color variation of ZIF-90 particle before and after adsorption of gaseous iodine. as ZIF-91 as a derivative of ZIF-90. These findings offer singleparticle visualization and mechanistic understanding of defectpromoted $\mathrm{I}_{2}$ adsorption in ZIF-90, thus proving the efficient avenue for revealing the structure-adsorption performance relationship.

\section{Results}

Visual observation of gaseous $I_{2}$ adsorption on single ZIF-90 particles. The ZIF-90 particles with a rhombic dodecahedron morphology and broad size distribution from 0.6 to $2.2 \mu \mathrm{m}$ are synthesized by the previously reported protocol (Fig. 2a, Supplementary Figs. 1 and 2) ${ }^{13}$. Powder XRD data confirms that the obtained patterns match the simulated one for ZIF-90, demonstrating the good purity of the sample (Supplementary Fig. 3). In order to evaluate the porosity, the $\mathrm{N}_{2}$ adsorption isotherm is measured at $77 \mathrm{~K}$ (Supplementary Fig. 4). The Brunauer-EmmettTeller (BET) area and pore size for ZIF-90 particles are calculated to be $897 \mathrm{~m}^{2} \cdot \mathrm{g}^{-1}$ and $18.7 \AA$, which are close to the reported values $^{14}$.

To image the gaseous $\mathrm{I}_{2}$ on single ZIF-90 particles, we dropcast the ZIF-90 dispersion on a microchamber, and the gaseous $I_{2}$ is pumped. As shown in Fig. 2b, single ZIF-90 particle can be resolved by DFM, showing a nearly white color, with a touch of blue. This is because the scattering spectrum of ZIF-90 particles displays a broad peak covering the whole visible-light region $(400-700 \mathrm{~nm})($ Fig. $2 \mathrm{c})$, which is similar to that of polystyrene (PS) microspheres with diameter of $10 \mu \mathrm{m}$ (the common whispering gallery-mode (WGM) resonators) (Supplementary Fig. 5) ${ }^{15-17}$. Recently, various MOFs have been confirmed to serve as WGM resonators as an alternative to PS microspheres ${ }^{18-20}$. Accordingly, the ZIF-90 particles are able to serve as WGM resonators for confining most of visible-light photons in their microstructures, resulting in a broad scattering peak (Supplementary Fig. 5a).

Fig. $2 \mathrm{~b}$ shows some snapshots of the DFM imaging movie at different reaction time (Supplementary movie 1). As the adsorption process of gaseous $\mathrm{I}_{2}$ on single ZIF-90 particle proceeds, the color of the edge of the particle begins to yellow at $30 \mathrm{~s}$. As the reaction time continues to increase, the entire ZIF90 particle surface turns to yellow at $90 \mathrm{~s}$. When the reaction time arrives at $180 \mathrm{~s}$, the color of the ZIF-90 particle will no longer be altered, corresponding to the maximum capture capacity of single ZIF-90 particle for gaseous $I_{2}$ (Fig. 2b). Correspondingly, the scattering spectrum of ZIF-90 particles after adsorption of $\mathrm{I}_{2}$ shows a significant intensity decrease in the blue light region with a wavelength around 400 to $525 \mathrm{~nm}$ (Fig. 2c), suggesting that the gaseous $\mathrm{I}_{2}$ can absorb the blue light, in line with the absorption spectrum of gaseous $I_{2}$ (Fig. 2d). Accordingly, the ZIF-90 particle after capture of $\mathrm{I}_{2}$ exhibits a yellow color based on the complementary color principle (the complementary color to blue is yellow). Also, we perform molecular dynamics (MD) simulation to study the gas diffusion and optical responses of the $\mathrm{I}_{2}$ loaded ZIF-90 particle. The calculated results suggest that ZIF-90 includes two independent $\mathrm{I}$ sites and a maximum of $8 \mathrm{I}_{2}$ molecules is accommodated inside each $\beta$ cage (Supplementary Fig. 6a). The smaller $\alpha$ cage is too constrained to allow for loading $\mathrm{I}_{2}$. The $\mathrm{I}_{2}$ molecules are highly mobile within the $\beta$ cage, but it is difficult that $\mathrm{I}_{2}$ molecules diffuse from one cage to another (Supplementary movie 2 and Supplementary Fig. 6b). Furthermore, the optical absorption properties of ZIF-90 with different $\mathrm{I}_{2}$ loading amounts are also calculated. As depicted in Supplementary Fig. 6c, pure ZIF-90 has no capacity for absorbing visible light, while the $\mathrm{I}_{2}$-loaded ZIF-90 can strongly absorb blue light (a wavelength between 400 and $525 \mathrm{~nm}$ ). Meanwhile, the higher $\mathrm{I}_{2}$ loading amount, the stronger absorption for blue light. These 
a

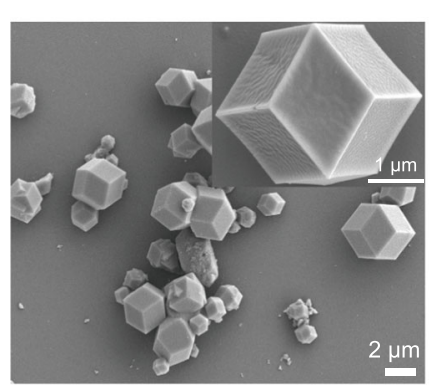

C

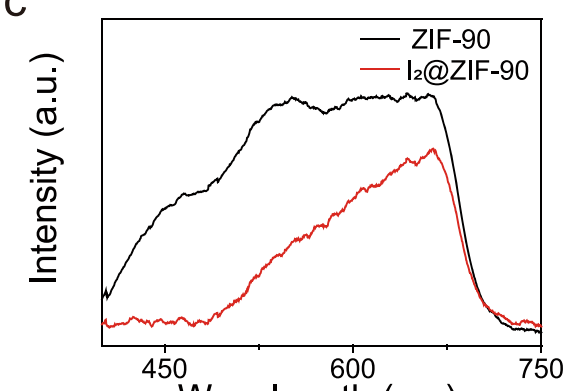

e

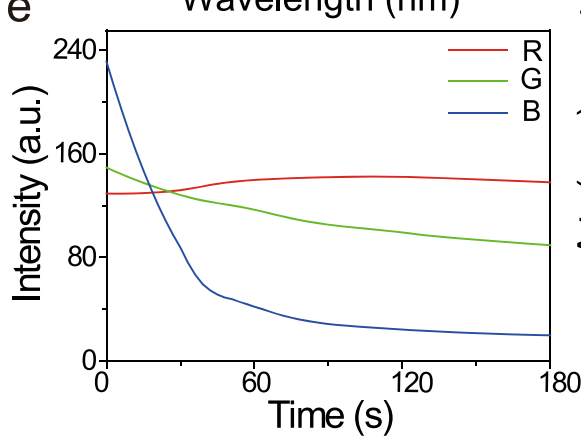

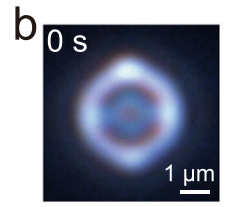
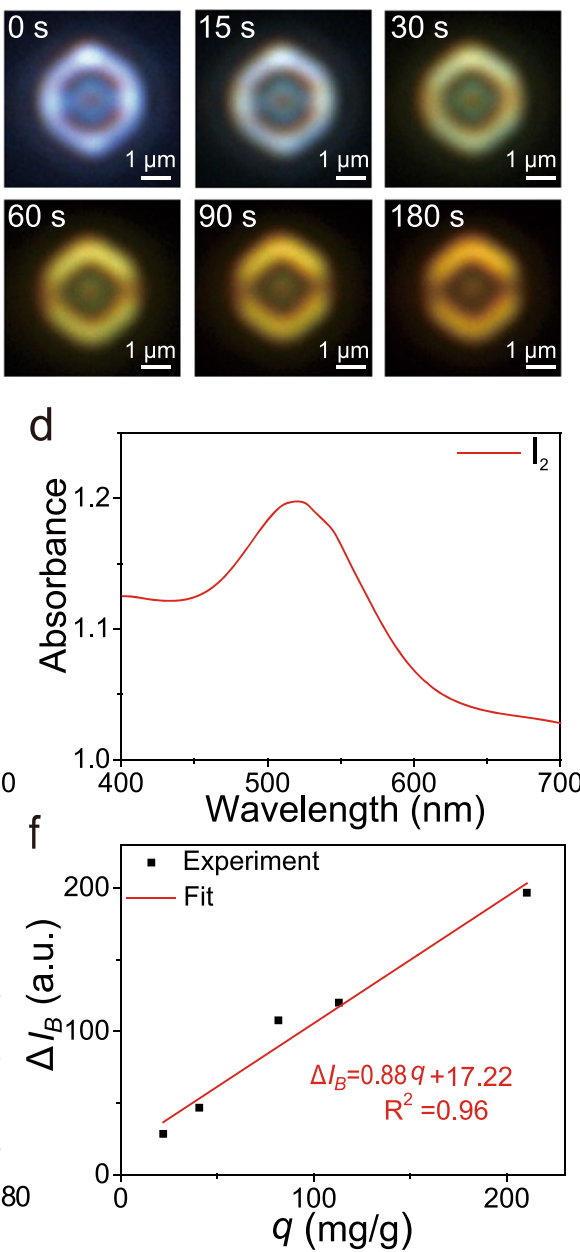

Fig. 2 Structure characterization of ZIF-90 particles and in situ DFM data of gaseous $\mathbf{I}_{\mathbf{2}}$ adsorption on single ZIF-90 particles. a SEM image of ZIF-90 particles. $\mathbf{b}$ Time-lapsed DFM images of gaseous $I_{2}$ adsorption on single ZIF-90 particles (The concentration of gaseous $I_{2}$ is $1 \mathrm{mM}$ ). c Scattering spectra of ZIF-90 particles before and after capturing $\mathrm{I}_{2}$. $\mathbf{d}$ The UV-vis adsorption spectrum of gaseous $\mathrm{I}_{2}$. e RGB values of DMF vs reaction time for capture of gaseous $I_{2}$ by single ZIF-90 particle. $\mathbf{f}$ Plot of the change of $B$ value $\left(\triangle I_{B}\right)$ from the DFM image as a function of adsorption amount of $I_{2}(q)$.

results are in accord with the experimental data as shown in Fig. 2b, c.

The successful adsorption of $\mathrm{I}_{2}$ by ZIF-90 particles is further demonstrated by several characterizations. First, most of the XRD peaks remain at similar $2 \theta$ positions but are weaken, indicating that the diffusion process of gaseous $\mathrm{I}_{2}$ retains the host crystallinity (Supplementary Fig. 3). Second, the BET surface area of ZIF-90 particles dramatically decreases to $4.38 \mathrm{~m}^{2} \cdot \mathrm{g}^{-1}$ after adsorption of $\mathrm{I}_{2}$ (Supplementary Fig. 4), mainly because of the filling of $\mathrm{I}_{2}$ molecules in the pores of ZIF-90. The elemental mapping analysis from the SEM image reveals the uniform distribution of $\mathrm{I}, \mathrm{C}, \mathrm{N}, \mathrm{O}$, and $\mathrm{Zn}$ elements in the surface of $\mathrm{I}_{2}$ loaded ZIF-90 particles (Supplementary Fig. 7). Besides, we employ the focused ion beam scanning electron microscopy (FIBSEM) to investigate the internal adsorption of $\mathrm{I}_{2}$. Supplementary Fig. 8 shows the SEM images of $\mathrm{I}_{2}$-loaded ZIF-90 slices created by FIB under different adsorption time and the corresponding elemental mapping of the cross section for $\mathrm{Zn}$ and I. It can be seen that a very uniform elemental distribution of $\mathrm{Zn}$ and $\mathrm{I}$ is found in the cross section of $\mathrm{I}_{2}$-loaded ZIF-90 microparticle. These results indicate that the adsorbed $\mathrm{I}_{2}$ is evenly distributed in ZIF-90 particle. The corresponding I/Zn molar ratio increases with the increase of the adsorption time from 0 to $180 \mathrm{~s}$ and then levels off when the adsorption time exceeds $180 \mathrm{~s}$ (Supplementary Fig. 9), suggesting that the adsorption of gaseous $I_{2}$ by ZIF-90 achieves an equilibrium state. Third, XPS studies are carried out to identify the chemical forms of $\mathrm{I}_{2}$. As depicted in Supplementary Fig. 10, the I $3 d$ peaks are decomposed into double peaks of $\mathrm{I}_{2}(632.1 \mathrm{eV}, 620.6 \mathrm{eV})$ and $\mathrm{I}_{3}{ }^{-}(630.4 \mathrm{eV}, 619 \mathrm{eV})^{21}$. The production of $\mathrm{I}_{3}{ }^{-}$proves the existence of the chemical adsorption and CT interaction between $\mathrm{I}_{2}$ and imidazole-2-carboxaldehyde $(\text { ICA })^{22}$. Additionally, the adsorbed $\mathrm{I}_{2}$ can be eluted from ZIF-90 particles with absolute EtOH, confirming that this adsorption process is reversible (Supplementary Fig. 11).

In addition, the DFM images of single ZIF-90 particles can be deconstructed into RGB chrominance information. As the color of the ZIF-90 particle from bluewhite to yellow, the B value of the DFM image decreases successively (Fig. 2e). The corresponding X ray/synchrotron data is provided by using transmission electron microscopy-energy-dispersive X-ray spectroscopy (TEM-EDS). Both $\triangle I_{B}$ and $\mathrm{I} / \mathrm{Zn}$ molar ratio follow a similar trend with increasing absorption time (Supplementary Fig. 12), revealing that the color change of the DFM image is induced by adsorption of gaseous $\mathrm{I}_{2}$ on ZIF-90 particles. We apply the variation of the $\mathrm{B}$ value $\left(\triangle I_{B}\right)$ to quantitatively estimate the adsorption of gaseous $\mathrm{I}_{2}$ on ZIF-90 particles via correlating $\triangle I_{B}$ with adsorption amount of $\mathrm{I}_{2}(q)$ (Supplementary Fig. 13 and Fig. 2f). The relationship between $\triangle I_{B}$ and $q$ can be fitted into a linear equation, $\triangle I_{B}=0.88 q+17.22$. Using this equation, we examine the adsorption kinetics of gaseous $\mathrm{I}_{2}$ on ZIF-90 particles. Fig. 3a 

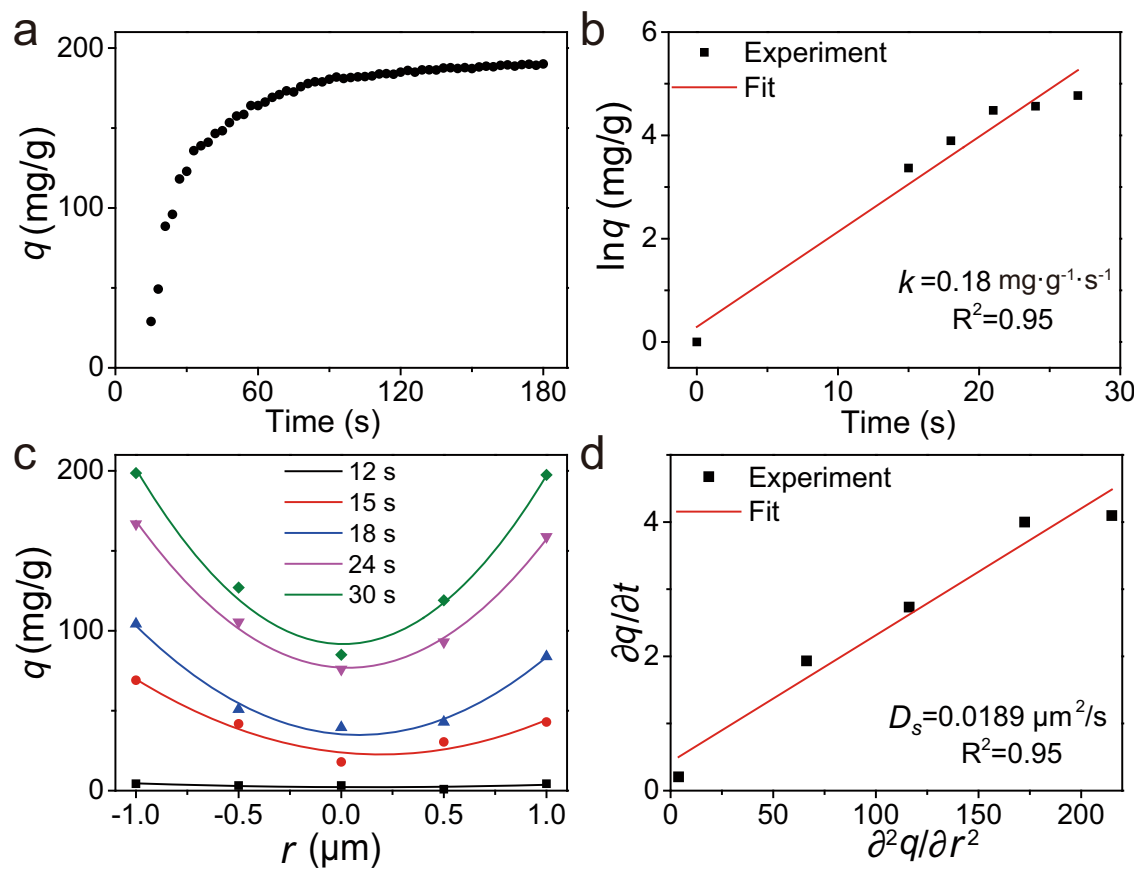

Fig. 3 Kinetic analysis and determination of diffusion coefficient. a Adsorption kinetic of gaseous $I_{2}$ onto a single ZIF-90 particle. $\mathbf{b}$ Pseudo-first order model for adsorption of gaseous $I_{2}$ to calculate the apparent rate constant $(k)$. c Spatial and temporal variability of $I_{2}$ absorption amount distributions in line profiles. d Diffusion coefficient $\left(D_{s}\right)$ estimated from Fick's second law.

shows the reaction time-dependence of $q$ from single ZIF-90 particle. There is a good linear correlation between $\ln q$ and reaction time, obeying pseudo-first order model (Fig. 3b), and the apparent rate constant $(k)$ is calculated to be $0.18 \mathrm{mg} \cdot \mathrm{g}^{-1} \cdot \mathrm{s}^{-1}$. Besides, under the assumption that the diffusivity coefficient $\left(D_{s}\right)$ is constant during the adsorption reaction, this process is capable of being described by Fick's second law:

$$
\frac{\partial C}{\partial t}=D_{s} \frac{\partial^{2} C}{\partial r^{2}}
$$

Where $t$ and $r$ are reaction time and the distance from the ZIF-90 particle center. By extracting spatial and temporal variability of gaseous $\mathrm{I}_{2}$ onto a single ZIF-90 particle in line profiles (Fig. 3c), the $D_{s}$ is estimated to be $0.0189 \mu \mathrm{m}^{2} / \mathrm{s}$ (Fig. 3d), which is comparable to that of other reported values ${ }^{23}$. Taken all together, this powerful DFM imaging approach can not only monitor the adsorption process of gaseous $\mathrm{I}_{2}$ on single ZIF-90 particle in real time and in situ, but also estimate the kinetic models and parameters as well as diffusion coefficients.

Interparticle heterogeneity in adsorption reactivity. The present DFM method permits simultaneously imaging the adsorption reactivity of multiple ZIF-90 particles. Fig. 4a is a series of DFM images of gaseous $\mathrm{I}_{2}$ adsorption on eight representative ZIF-90 particle obtained in situ, labeled with P1 to P8. Although all the eight ZIF-90 particles gradually undergo a color change from bluewhite to yellow within $180 \mathrm{~s}$, the change magnitudes and rates of the $\mathrm{B}$ value are distinct, indicating different adsorption amount and dynamic toward gaseous $I_{2}$ for different ZIF-90 particles. For example, P1-P5, and P7 firstly approach to adsorption equilibrium at $90 \mathrm{~s}$, followed by P6 and P8 (Fig. 4b). The adsorption amount of P2 for gaseous $\mathrm{I}_{2}$ is about $226 \mathrm{mg} / \mathrm{g}$, which is much higher than that of P8 $(\sim 150 \mathrm{mg} / \mathrm{g})$. Further statistical absorption amounts of 30 individual ZIF-90 particles reveal a significant particle-to-particle variation (Supplementary Fig. 14), and a 2.26 -fold variation on the adsorption amounts of single ZIF-90 particles is found as shown in Fig. 4c. These data demonstrate the interparticle heterogeneity in the adsorption reactivity for gaseous $I_{2}$. This level of information in the adsorption process is impossible to be achieved by using conventional spectral measurement on bulk sample.

It is generally assumed that the heterogeneity is related to the particle size. Taking P6 and P7 for example (Fig. 4b), P7 with a large size $(3.8 \mu \mathrm{m})$ exhibits faster adsorption kinetic and more adsorption amount $(q=220 \mathrm{mg} / \mathrm{g})$ than that of P6 with a small size $(1.8 \mu \mathrm{m})(q=151 \mathrm{mg} / \mathrm{g})$. However, the size of P7 is very similar to P8 $(3.6 \mu \mathrm{m})$, but the adsorption capacity of P7 is strikingly different from P8 $(q=150 \mathrm{mg} / \mathrm{g})$ as illustrated in Fig. 4 b. Therefore, the particle size of the ZIF-90 particles is not remarkably correlated with the adsorption amount for gaseous $\mathrm{I}_{2}$. This assumption is further supported via correlation analysis with a Pearson correlation coefficient of +0.42 (Fig. $4 \mathrm{~d}$ ), meaning that the particle size and adsorption amount have no strong relationship with each other. Previous fluorescence microscopy imaging studies infer that the heterogeneity of MOFs is attributed to the interior defect level caused by fluctuations in the preparation conditions $^{24,25}$. The imperfect crystal structure of ZIF-90 particles are testified by SEM image (Fig. 2a). Some ZIF-90 particles have very regular rhombic dodecahedron shape, implying the good crystallinity, while others display serious crystal dislocation and rough surface (Fig. 2a). These results suggest that the heterogeneity of ZIF-90 particles in adsorption reactivity for gaseous $\mathrm{I}_{2}$ is dominated by particle-to-particle defect level differences.

Defect-dominated adsorption performance of single ZIF-90 particles. In addition to investigating the interparticle heterogeneity, another noticeable feature of this imaging method is that it permits for evaluating the adsorption performance of defected ZIF-90 at the single-particle level. The inherent defect of ZIF-90 is relatively scarce, and water postsynthetic treatment is validated to be an efficient avenue for introducing more defects into ZIF-90 

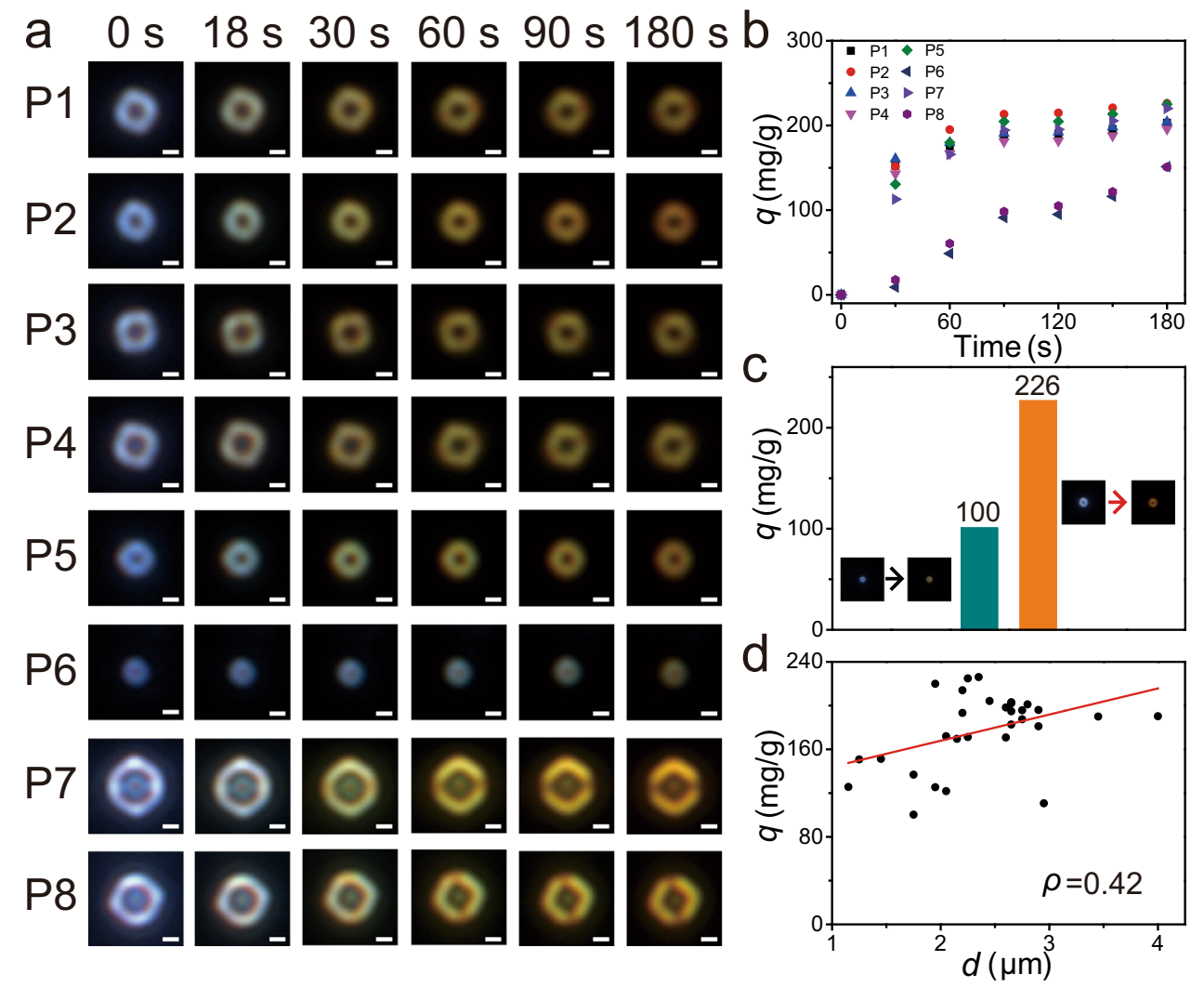

Fig. 4 Imaging the gaseous $\mathbf{I}_{\mathbf{2}}$ adsorption on ZIF-90 particles. a Time-resolved DFM images of gaseous $I_{2}$ adsorption on eight representative ZIF-90 particles (scale bar: $1 \mu \mathrm{m}$ ) and (b) the corresponding adsorption kinetic. c Comparison of the absorption amount ( $q$ ) between two ZIF-90 particles. The inset shows the DFM image of the ZIF-90 particles before and after adsorption of $\mathrm{I}_{2}$. $\mathbf{d}$ Correlation plot and the corresponding Pearson's correlation coefficient $(\rho)$ between absorption amount and diameter of ZIF-90 particles.

according to the generation of linker defects in the framework (Supplementary Fig. 15) ${ }^{26}$. The structure characterizations of the defected ZIF-90 before and after adsorption of gaseous $I_{2}$ are depicted in Supplementary Figs. 16-19. Using the time sequential DFM images, we then quantify the kinetics of six representative defected ZIF-90 particles (Fig. 5), marked with P\#1 to P\#6. It is observed that the adsorption rate of defected ZIF-90 particles increases considerably. For instance, all the six defected ZIF-90 particles can attain adsorption chemical equilibrium within $60 \mathrm{~s}$, which is apparently shorter than that of ZIF-90 particles (Figs. 5b and $4 \mathrm{~b})$. The statistical average value of the apparent rate constant for defected ZIF-90 particles is improved twice as much as that of the primary ZIF-90 particles (Supplementary Figs. 20 and 21). The same trends occur for the increase in the adsorption amount of $\mathrm{I}_{2}$ and diffusivity coefficient (Fig. $5 \mathrm{c}-\mathrm{d}$, and Supplementary Figs. 20-21). These data confirm that the defects in ZIF-90 particles play an important role in the adsorption of $I_{2}$, which can increase adsorption rate, adsorption amount, and diffusivity coefficient.

In general, the CT interaction between electron-deficient $\mathrm{I}_{2}$ molecule and electron-rich aromatic ring of the imidazole is responsible for the capture of $\mathrm{I}_{2}$ by ZIF-90 particles (Supplementary Fig. 23) $)^{9,11}$. In the ZIF-90, the electron configuration of $\mathrm{Zn}^{2+}$ is $[\operatorname{Ar}] 3 d^{10}$, and the $4 s$ and three $4 p$ orbitals $\left(p_{x}, p_{y}\right.$, and $\left.p_{z}\right)$ hybridize to generate four vacant $s p^{3}$ orbitals, which coordinate with the unpaired electron on the nitrogen atoms of the ICA to form $\mathrm{Zn}-\mathrm{N}$ coordinate covalent bonds (Fig. 5e). This coordination interaction usually results in the delocalization of the electrons, decreasing the electron density of the imidazole ring. To depict the change of the electron density, we analyze the electrostatic potential (ESP) of ICA before and after coordination with $\mathrm{Zn}^{2+}$.
The results, as depicted in Supplementary Fig. 24, clearly indicate that the $\mathrm{Zn}^{2+}$-ICA complex is more electron deficient as compared to ICA. However, when the linker defects are introduced, partial $\mathrm{Zn}-\mathrm{N}$ bonds will be broken (Supplementary Fig. 15), which alleviates the delocalization of $\mathrm{n}$ electrons in $\mathrm{N}$ atoms from ICA, leading to the increase of the electron density. Owing to the high electron density of the defected ZIF-90, it favors the CT interaction between $\mathrm{I}_{2}$ and ICA aromatic ring, promoting the adsorption of $\mathrm{I}_{2}$ on ZIF-90 particles. Additionally, it also should be noted that we can not completely exclude the influence of the potential interference from grain boundary issues in the particles or purity of crystallinity on the adsorption of gaseous $\mathrm{I}_{2}$ due to the large particle-to-particle heterogeneity.

Visual observation of gaseous $I_{2}$ adsorption on single ZIF-91 particles. As an illustration of the generality of this imaging method for studying in situ the adsorption of $\mathrm{I}_{2}$, another ZIF material, ZIF-91 that is synthesized by reduction of ZIF-90 using $\mathrm{NaBH}_{4}$ is investigated as well (Supplementary Fig. 25). The XRD pattern, SEM image and elemental mapping demonstrate the successful preparation of ZIF-91 with the capacity of capturing $\mathrm{I}_{2}$ (Fig. 6a, Supplementary Figs. 26 and 27). Fig. 6b shows the timeresolved DFM images of gaseous $\mathrm{I}_{2}$ adsorption on six representative ZIF-91 particles, labeled with P1' to P6'. Apart for P4', the corners of other five ZIF-91 particles start to become yellow at $\sim 21 \mathrm{~s}$. After reaction for $90 \mathrm{~s}$, the $\triangle I_{B}$ values of all the five ZIF91 particles level off, corresponding to the adsorption equilibrium state (Fig. 6c). The large heterogeneity in the adsorption kinetic of individual particles is also observed as displayed in Fig. $6 \mathrm{c}$ and Supplementary Fig. 28. These observations are consist with the 

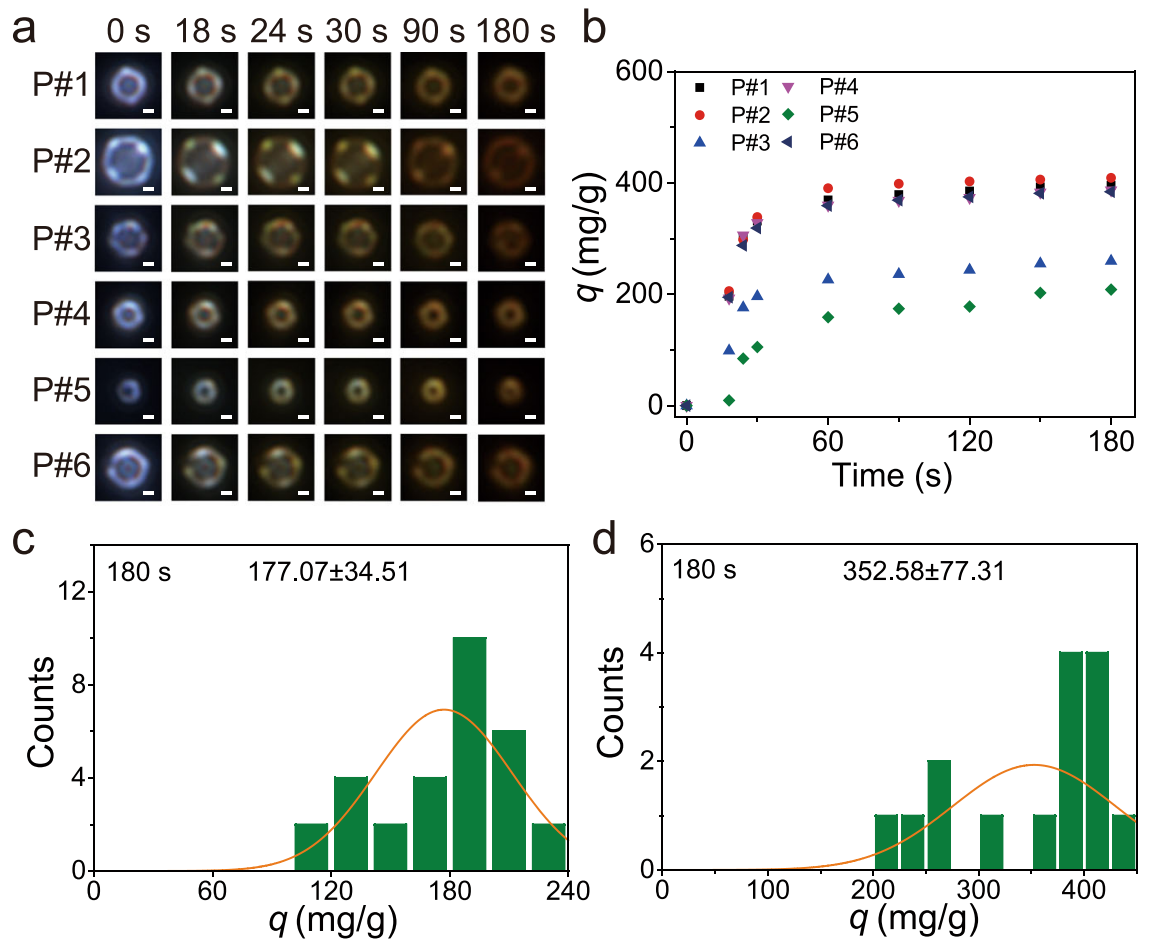

e

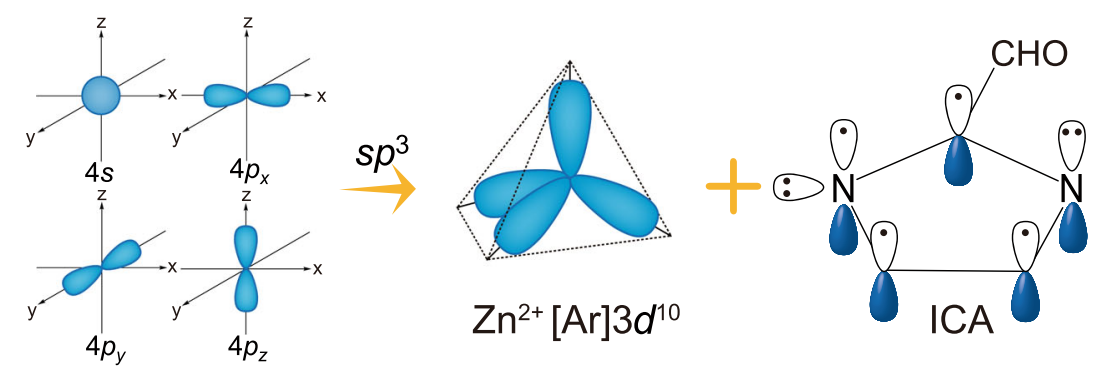

Fig. 5 Visualization of gaseous $\mathbf{I}_{\mathbf{2}}$ adsorption on defected ZIF-90 particles. a Time sequential DFM images of gaseous $I_{2}$ adsorption on six representative defected ZIF-90 particles (scale bar: $1 \mu \mathrm{m}$ ) and (b) the corresponding adsorption kinetic. Histograms of the adsorption amount ( $q$ ) for (c) ZIF-90 and (d) defected ZIF-90 particles. e Formation of $s p^{3}$ orbitals from $4 s$ and $4 p$ orbitals of $\mathrm{Zn}^{2+}$ and molecular orbital structure of ICA.
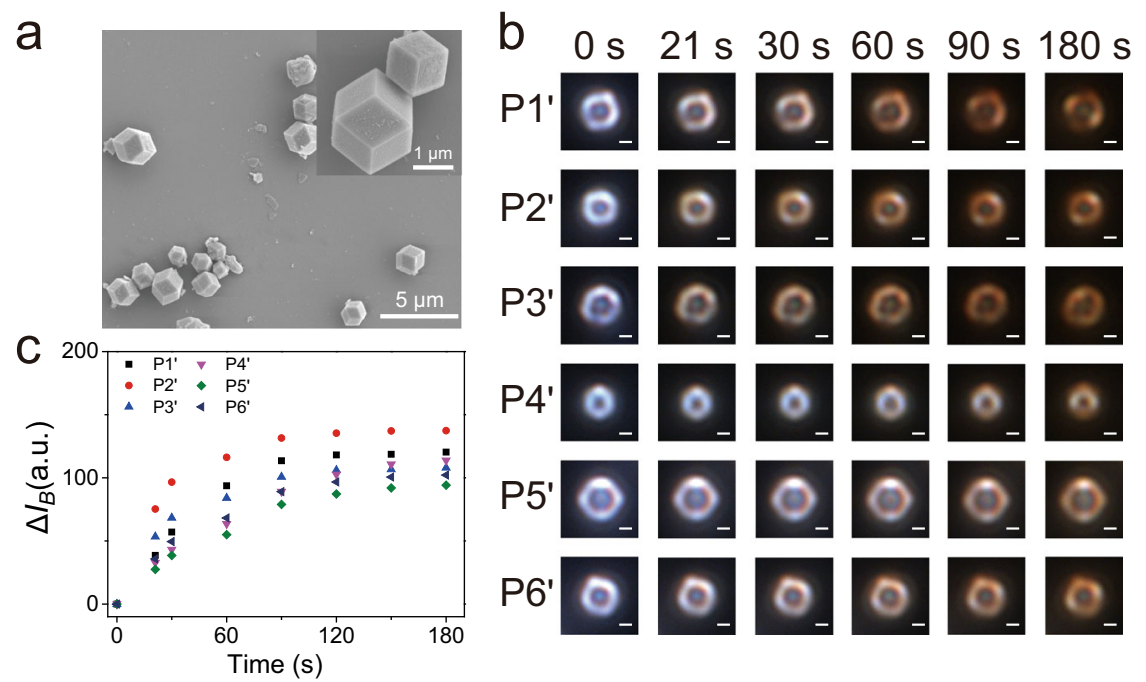

Fig. 6 Visually monitoring gaseous $\mathbf{I}_{\mathbf{2}}$ adsorption on individual ZIF-91 particles. a SEM image of ZIF-91 particles. $\mathbf{b}$ Time-resolved DFM images of gaseous $I_{2}$ adsorption on six representative ZIF-91 particles (scale bar: $1 \mu \mathrm{m}$ ) and $\left(\mathbf{c}\right.$ ) the corresponding adsorption kinetic. $\triangle I_{B}$ represents the change of $B$ value of the DFM images. 
reaction trends obtained in the ZIF-90 particles. Overall, the present imaging strategy is extremely suitable to investigate the $\mathrm{I}_{2}$ adsorption by various ZIF materials.

\section{Discussion}

In conclusion, we have demonstrated that the DFM method allows the visualization of gaseous $\mathrm{I}_{2}$ adsorption on single ZIF-90 particles. By correlating the $\triangle I_{B}$ with adsorption amount of $\mathrm{I}_{2}$, we can quantitatively determine the adsorption rate, adsorption amount, and diffusivity coefficient at the single particle level. DFM image analysis reveals the large heterogeneity in adsorption activity for $\mathrm{I}_{2}$ among different ZIF-90 particles because of the distinct particle-to-particle defect level. In addition, the present imaging method permits us to compare the adsorption performance of ZIF-90 particles before and after introduction of linker defects. The experimental results show that the linker defects result in the electron density increase of the imidazole ring, greatly promoting the adsorption of $\mathrm{I}_{2}$ on the defected ZIF-90 particles. Furthermore, the generality of this imaging method is also affirmed by showing the capacity for studying gaseous $\mathrm{I}_{2}$ adsorption on single ZIF-91 particles. This strategy using in situ DFM approach provides a fundamental understanding of the structure-performance relationship, which is potential to guide rational design and development of high-efficient porous MOF adsorbents.

\section{Methods}

Materials. Imidazole-2-carboxaldehyde, polyvinylpyrrolidone, 2-methylimidazole, and sodium borohydride are obtained from Aladdin Chemical Reagent Co., Ltd. Iodine is purchased from Sangon Co., Ltd. Zinc nitrate hexahydrate, tert-butyl alcohol, n-hexane, methanol, and ethanol are obtained from Chengdu Kelong Chemicals Co., Ltd.

Preparation of ZIF-90 particles. The ZIF-90 particles are prepared by a simple one-pot method ${ }^{13}$. Briefly, $0.02 \mathrm{~g}$ polyvinylpyrrolidone and $0.12 \mathrm{~g}$ imidazole-2carboxaldehyde are dissolved into $9 \mathrm{~mL}$ of deionized water, which is stirred for 3 min at $25^{\circ} \mathrm{C}$. After that, $10 \mathrm{~mL}$ of $0.033 \mathrm{mM}$ zinc nitrate solution dissolved in tertbutyl alcohol is injected into the above solution under vigorous stirring for $10 \mathrm{~min}$ at $30^{\circ} \mathrm{C}$. The ZIF-90 particles are collected by centrifugation $(3600 \times g$ for $2 \mathrm{~min})$, washed three times with methanol, and resuspended in absolute alcohol.

Preparation of defected ZIF-90 particles induced by water treatment. For preparation of defected ZIF- 90 particles ${ }^{27}, 5 \mathrm{mg}$ ZIF- 90 is added to $5 \mathrm{~mL}$ deionized water. Subsequently, the solution is heated to $50{ }^{\circ} \mathrm{C}$ maintained at this temperature for $1 \mathrm{~h}$, and the defected ZIF-90 particles are collected by centrifugation $(3600 \times g$ for $2 \mathrm{~min}$ ) and dried at $70^{\circ} \mathrm{C}$ for $12 \mathrm{~h}$.

Preparation of ZIF-91 particles. The ZIF-91 particles are synthesized by reduction of ZIF-90 by $\mathrm{NaBH}_{4}{ }^{14}$. ZIF-90 particles $(0.1 \mathrm{~g}$ ) are dispersed in $10 \mathrm{~mL}$ of $1.56 \mathrm{mM}$ $\mathrm{NaBH}_{4}$ methanol solution and refluxed for $24 \mathrm{~h}$. Then, the reaction solution is filtered. The resulting solid sample is washed twice with methanol, which is further exchanged with methanol for 1 day to obtain ZIF-91 particles. Then, the prepared ZIF-91 is dried at $70^{\circ} \mathrm{C}$ for $12 \mathrm{~h}$.

Single-particle imaging of gaseous $\mathbf{I}_{\mathbf{2}}$ adsorption. The imaging of gaseous $\mathrm{I}_{2}$ adsorption on single ZIF-90 particles is performed using Olympus BX53 optical microscopy setup with a white light source (halogen lamp, $100 \mathrm{~W}$ ), dark-field condenser (numerical aperture: 1.2-1.4), chip true-color charge-coupled device camera, and 100x immersion objective (numerical aperture: 0.75). The ZIF-90 particles dissolved in ethanol solution are drop-casted onto a glass slide, and dried in air. Then, a borosilicate coverslip and two micropipes are covered on the surface of the glass slide, and sealed by double-sided tape to fabricate a microchamber $(2$ $\mathrm{cm} \times 1.5 \mathrm{~cm} \times 1.5 \mathrm{~mm}$ ). Soon after, the gaseous $\mathrm{I}_{2}$ with a concentration of $1 \mathrm{mM}$ is successively pumped into the reactor cell, and the excessive gaseous $\mathrm{I}_{2}$ is adsorbed by ethanol. The scattering spectra and DFM image are collected by a fiber optic spectrometer (EK2000, Shanghai Choptics).

For imaging the gaseous $\mathrm{I}_{2}$ adsorption on single defected ZIF-90 and ZIF-91 particles, the same experimental operations are performed.

Characterization. The morphological characteristics of the ZIF particles are investigated by using Zeiss Merlin field-emission transmission electron microscope (TEM, Libra200, accelerating voltage: $200 \mathrm{kV}$ ) and scanning electron microscope
(SEM, UItra55). Elemental distribution diagram is collected by energy dispersive $\mathrm{X}$ ray spectroscopy (EDS) connected with field-emission SEM. The crystal structures are studied by X' Pert Pro X-ray diffractometer. Specific surface area of the ZIF particles are collected from nitrogen physisorption isotherms at $77 \mathrm{~K}$. The specific surface area and pore size distribution are calculated with Saito-Foley (SF) model ${ }^{28}$ by employing an Autosorb iQ-MP/XR gas sorption analyzer (Quantachrome instruments). The chemical compositions of the samples are investigated by ESCALAB 250Xi X-ray photoelectron spectrometer (XPS), and Shimadzu UV-1900 UV/VIS spectrometer.

\section{Data availability}

The data that support the findings detailed in this study are available in the Article and its Supplementary Information or from the corresponding authors upon reasonable request. Source data are provided with this paper.

Received: 28 March 2021; Accepted: 6 July 2021; Published online: 23 July 2021

\section{References}

1. Wang, P. et al. Exceptional iodine capture in 2D covalent organic frameworks. Adv. Mater. 30, 1801991 (2018).

2. Saiz-Lopez, A. et al. Atmospheric chemistry of iodine. Chem. Rev. 112, 1773-1804 (2012).

3. Burns, P. C., Ewing, R. C. \& Navrotsky, A. Nuclear fuel in a reactor accident. Science 335, 1184-1188 (2012).

4. Konings, R. J., Wiss, T. \& Beneš, O. Predicting material release during a nuclear reactor accident. Nat. Mater. 14, 247-252 (2015).

5. Iglesias-Suarez, F. et al. Natural halogens buffer tropospheric ozone in a changing climate. Nat. Clim. Change 10, 147-154 (2020).

6. Baccarini, A. et al. Frequent new particle formation over the high Arctic pack ice by enhanced iodine emissions. Nat. Commun. 11, 4924 (2020).

7. Piatkowski, L., Zhang, Z., Backus, E. H., Bakker, H. J. \& Bonn, M. Extreme surface propensity of halide ions in water. Nat. Commun. 5, 4083 (2014).

8. Tham, Y. J. et al. Direct field evidence of autocatalytic iodine release from atmospheric aerosol. Proc. Natl Acad. Sci. USA. 118, e2009951118 (2021).

9. Hughes, J. T., Sava, D. F., Nenoff, T. M. \& Navrotsky, A. Thermochemical evidence for strong iodine chemisorption by ZIF-8. J. Am. Chem. Soc. 135 , 16256-16259 (2013)

10. Sava, D. F. et al. Capture of volatile iodine, a gaseous fission product, by zeolitic imidazolate framework-8. J. Am. Chem. Soc. 133, 12398-12401 (2011)

11. Zeng, M.-H. Rigid pillars and double walls in a porous metal-organic framework: single-crystal to single-crystal, controlled uptake and release of iodine and electrical conductivity. J. Am. Chem. Soc. 132, 2561-2563 (2010).

12. Chapman, K. W., Sava, D. F., Halder, G. J., Chupas, P. J. \& Nenoff, T. M. Trapping guests within a nanoporous metal-organic framework through pressure-induced amorphization. J. Am. Chem. Soc. 133, 18583-18585 (2011)

13. Zhang, J. et al. Colorable zeolitic imidazolate frameworks for colorimetric detection of biomolecules. Anal. Chem. 92, 12670-12677 (2020).

14. Morris, W., Doonan, C. J., Furukawa, H., Banerjee, R. \& Yaghi, O. M. Crystals as molecules: postsynthesis covalent functionalization of zeolitic imidazolate frameworks. J. Am. Chem. Soc. 130, 12626-12627 (2008).

15. He, L., Özdemir, Ş. K., Zhu, J., Kim, W. \& Yang, L. Detecting single viruses and nanoparticles using whispering gallery microlasers. Nat. Nanotechnol. 6 , 428-432 (2011).

16. Venkatakrishnarao, D., Mamonov, E. A., Murzina, T. V. \& Chandrasekar, R. Advanced organic and polymer whispering-gallery-mode microresonators for enhanced nonlinear optical light. Adv. Opt. Mater. 6, 1800343 (2018).

17. Smith, M. J. et al. Coupled whispering gallery mode resonators via templateassisted assembly of photoluminescent microspheres. Adv. Funct. Mater. 29, 1902520 (2019).

18. $\mathrm{He}, \mathrm{H}$. et al. Polarized three-photon-pumped laser in a single MOF microcrystal. Nat. Commun. 7, 11087 (2016).

19. Lv, Y. et al. Pure metal-organic framework microlasers with controlled cavity shapes. Nano Lett. 20, 2020-2025 (2020).

20. Gao, Z. et al. Topological distortion driven amorphous spherical MOFs for high quality single-mode microlasers. Angew. Chem. Int. Ed. 60, 6362-6366 (2021).

21. Chen, L. et al. Mesoporous zeolitic imidazolate framework-67 nanocrystals on siliceous mesocellular foams for capturing radioactive iodine. ACS Appl. Nano Mater. 3, 5390-5398 (2020).

22. Wang, Z. et al. The water-based synthesis of chemically stable Zr-based MOFs using pyridine-containing ligands and their exceptionally high adsorption capacity for iodine. Dalton Trans. 46, 7412-7420 (2017). 
23. Hendriks, F. C. et al. Single-molecule fluorescence microscopy reveals local diffusion coefficients in the pore network of an individual catalyst particle. $J$. Am. Chem. Soc. 139, 13632-13635 (2017).

24. Ameloot, R. et al. Three-dimensional visualization of defects formed during the synthesis of metal-organic frameworks: a fluorescence microscopy study. Angew. Chem. Int. Ed. 125, 419-423 (2013).

25. Schrimpf, W. et al. Chemical diversity in a metal-organic framework revealed by fluorescence lifetime imaging. Nat. Commun. 9, 1647 (2018).

26. Fang, Z., Bueken, B., De Vos, D. E. \& Fischer, R. A. Defect-engineered metalorganic frameworks. Angew. Chem. Int. Ed. 54, 7234-7254 (2015).

27. Zhang, C., Han, C., Sholl, D. S. \& Schmidt, J. R. Computational characterization of defects in metal-organic frameworks: Spontaneous and water-induced point defects in ZIF-8. J. Phys. Chem. Lett. 7, 459-464 (2016).

28. Saito, A. \& Foley, H. C. Curvature and parametric sensitivity in models for adsorption in micropores. AIChE J. 37, 429-436 (1991).

\section{Acknowledgements}

The support of this research by the National Natural Science Foundation of China (21705134) is gratefully acknowledged. The authors appreciate Dr. Zhenwei Niu and Dr. Tong Liu from Southwest University of Science and Technology and Suzhou Institute of Nano-Tech and Nano-Bionics (Chinese Academy of Sciences) for the theoretical calculations and FIB-SEM measurement.

\section{Author contributions}

Y.H. and H.L.Y. conceived and designed the research. Y.T.L., G.H.Z., Q.L.Z., and L.Y conducted the experiments. H.L. performed material characterization experiments. Al authors analyzed the results and commented on the manuscript.

\section{Competing interests}

The authors declare no competing interests.

\section{Additional information}

Supplementary information The online version contains supplementary material available at https://doi.org/10.1038/s41467-021-24830-1.

Correspondence and requests for materials should be addressed to H.Y. or Y.H.

Peer review information Nature Communications thanks the anonymous reviewers for their contributions to the peer review of this work.

Reprints and permission information is available at http://www.nature.com/reprints

Publisher's note Springer Nature remains neutral with regard to jurisdictional claims in published maps and institutional affiliations.

(c) (i) Open Access This article is licensed under a Creative Commons Attribution 4.0 International License, which permits use, sharing, adaptation, distribution and reproduction in any medium or format, as long as you give appropriate credit to the original author(s) and the source, provide a link to the Creative Commons license, and indicate if changes were made. The images or other third party material in this article are included in the article's Creative Commons license, unless indicated otherwise in a credit line to the material. If material is not included in the article's Creative Commons license and your intended use is not permitted by statutory regulation or exceeds the permitted use, you will need to obtain permission directly from the copyright holder. To view a copy of this license, visit http://creativecommons.org/ licenses/by/4.0/.

(C) The Author(s) 2021 